\title{
Can Multi-Stage Recombinant Fusion Proteins be Considered as Reliable Vaccines Against Tuberculosis? A Letter to the Editor
}

\author{
Masoud Keikha (iD) ${ }^{1}$ and Mohsen Karbalaei (iD) ${ }^{2, *}$ \\ ${ }^{1}$ Department of Microbiology and Virology, Faculty of Medicine, Mashhad University of Medical Sciences, Mashhad, Iran \\ ${ }^{2}$ Department of Microbiology and Virology, School of Medicine, Jiroft University of Medical Sciences, Jiroft, Iran \\ "Corresponding author: Department of Microbiology and Virology, School of Medicine, Jiroft University of Medical Sciences, Jiroft, Iran. Email: \\ mohsenkarbalaei691@gmail.com
}

Received 2019 March 11; Revised 2019 April 30; Accepted 2019 May 03.

Keywords: Tuberculosis, Vaccine, Multi-Stage Vaccine

\section{Dear Editor,}

Tuberculosis (TB) caused by Mycobacterium tuberculosis complex (MTBC) remains a major global concern, especially in developing countries. According to the World Health Organization (WHO) reports, 1.4 million deaths occurred among all 10.4 million TB cases in 2016 (1).

Unfortunately, the emergence and spread of multidrug resistant tuberculosis (MDR-TB) strains, which are resistant to two most powerful anti-TB drugs including rifampin and isoniazid, and extensively-drug resistant tuberculosis (XDR-TB) strains, which are resistant to any fluoroquinolone in addition to rifampin and isoniazid, have become major therapeutic challenges. Moreover, co-infection with TB and human immunodeficiency virus (HIV) should be considered as a critical alarm for failure in universal directly observed treatment short-course (DOTS) strategies and TB control programs. Approximately 600,000 new cases of rifampin mono-resistant and MDRTB emerged around the world in 2016, leading to 240,000 deaths in $2017(1,2)$.

Nowadays, given the current state of tuberculosis, the most useful strategies for controlling and prevention of this global health problem include the detection of patients with active TB using smear screening, treatment of patients, stopping and combating HIV, introducing novel drugs, controlling the emergence of drug-resistant TB, TB vaccination, and detection/treatment of latent tuberculosis that includes one-third of the population contaminated by M. tuberculosis (Mtb) with no clinical symptoms. Patients with latent tuberculosis are the sources of infection while only $5 \%-15 \%$ of them develop to the active form of tuberculosis (2,3).
Vaccination is the main strategy for the protection, immunization, and stopping of infectious diseases. At present, the attenuated strain of Mycobacterium bovisbacillus Calmette-Guerin, called the BCG vaccine, has been the only available recommended vaccine against tuberculosis since 1921. Although the BCG vaccine is effective for the protection against miliary and meningitis TB forms in children, its effect is highly variable $(0 \%-80 \%)$ against pulmonary tuberculosis (PTB) in adults and latent tuberculosis patients. Moreover, the BCG vaccine is associated with side effects such as lupoid reaction, non-suppurative lymphadenitis, osteomyelitis, and disseminated BCG infection in HIV patients. The BCG vaccine is also not recommended for patients with immune disorders such as AIDS. Therefore, one of the most challenging issues of tuberculosis is the development of a novel vaccine that is useful for all groups, including immune disorder patients and healthy individuals, and creates an effective immune response against latent tuberculosis $(4,5)$.

Today, there are numerous tuberculosis vaccine candidates such as recombinant BCG (rBCG), the attenuated mutants of $M t b$, multi-stage fusion peptides, viral vector vaccines, DNA vaccines, and protein subunit vaccines. Overall, TB candidate vaccines are classified into three categories including (1) pre-exposure or prophylactic candidates recommended for the induction of strong cellular immunity against TB before infection, (2) post-exposure candidates administered for the eradication of $M t b$ infection (latentTB infection) or prevention of TB reactivation, and (3) therapeutic candidates for the treatment of active TB patients and MDR-TB, XDR-TB, or extremely-drug resistant TB (XXDR$\mathrm{TB})(6,7)$. The $M t b$ bacterium is an aerobic facultative in- 
tracellular bacillus. The cell-mediated immune (CMI) system plays a key role in the limitation of $M t b$ replication in macrophages; it contributes in controlling of TB infection by the production of immune cytokines, such as TNF$\alpha$ and IFN- $\gamma$, and the activation of macrophages for the elimination of TB bacilli or directly lysis of TB bacilli reservoirs (alveolar macrophages). According to a review of the literature, subunit vaccines participate in the activation of the immune system response against $M t b$ infection through $\mathrm{T}$ cells differentiation, macrophage stimulation, or immune cytokines production, particularly IFN- $\gamma$ (5). One of the main disadvantages of the BCG vaccine is the absence of strong-immunological memory cells, causing the lack of BCG immunity during latent-TB infection and consequently, TB reactivation (8). Several experimental studies proposed that subunit vaccines could provoke the central immunological memory cells, which continuously produced IL-2 for T effector cells proliferation against tuberculosis and maintained over one year after vaccination $(8,9)$.

Several types of these vaccine candidates, particularly subunit vaccines, have been developed are investigated in preclinical or clinical trials, such as: H64+CAF01 (consisting of six immunogenic $M t b$ antigens including ESAT6, EspD, EspC, EspF, EspR, and PE35 along with CAF10 as a liposomal adjuvant) and rBCG $\Delta$ ais1/zmp1 (recombinant BCG with deletion of the $z m p 1$ gene) as vaccine candidates in preclinical studies; MVA85A (a recombinant Modified Vaccinia Ankara (MVA) virus which expresses high levels of Ag85A), TB/FLU-04L (a recombinant influenza virus H1N1 with two main antigens of Mtb, ESAT-6 and Ag85B), and Ad5Ag85A (a recombinant non-replicating adenovirus serotype 5 expressing Ag85B) as TB vaccine candidates in clinical trials phase I (10); RUTI (fragmented $M t b$ ), H1/H56:IC31 (a subunit vaccine containing Ag85B and ESAT6 with IC31 as adjuvant), and ID93+GLA-SE (containing Rv2608, Rv3619, Rv1813, Rv3620, and a synthetic lipid adjuvant) entering phase IIa clinical trials; DAR-901 (wholecell Mycobacterium obuense) and M72+AS01E (PPE18 and PepA mycobacterial peptides with a liposomal adjuvant) in phase IIb clinical trials; and finally, Mycobacterium vaccae (whole-cell killed M.vaccae) for treatment of pulmonary tuberculosis, leprosy, and MDR-TB.

Several novel TB subunit peptide vaccines have entered the various stages of clinical trials or preclinical experiments. Candidates including M72+AS01E, H1/H56:IC31, H4:IC31, ID93+GLASE, and H64+CAF10 are the subunit peptide vaccines that are passing phases I, IIa, and IIb clinical trials. Fusion subunit peptides are usually designed based on early antigens released by replicating TB bacilli for the prevention of active tuberculosis, such as ESAT-6, CFP-10, Ag85B, and TB 10.4, which are located on the RD1 locus, as a locus that does not exist on M. bovis, M. bovis BCG, and M. microti. In addition, these fusion peptides should have latent $M t b$ antigens such as HspXor DosR in their structures for the prevention of latent tuberculosis. As CMI and Th1 cells play important roles in stopping $\mathrm{TB}$, in some studies, the Fc domains of antibodies (particularly IgG1) are attached to this subunit peptide for the enhancement of immune delivery system, selective uptake by antigen presentation cells (APCs), cross-priming of macrophages, and increasing the half-life and solubility of this component. According to the literature, the H1:IC31 fusion peptide can promote immunity in healthy volunteers and latent tuberculosis (HIV negative) patients without side effects. This peptide can induce multifunctional $\mathrm{CD} 4^{+} \mathrm{T}$ cell responses in previous healthy recipients of the BCG vaccine. H56:IC31 could also induce long-term immunity in non-human primate models.

ID93:GLA-SE induces multi-functional Th1 cells against Beijing strains of $M t b$ through the production of IL-2, TNF- $\alpha$, and IFN- $\gamma$ in TB-infected human hosts. Moreover, M72+AS01E produces a strong immune response (both humoral and cellular immunity) in all age groups without side effects (Table 1) $(4-6,11)$.

Multi-stage subunit TB candidate vaccines constitute a large portion of TB candidate vaccines, which are used for various applications, such as pre-exposure and postexposure vaccination, or even therapeutic purposes, including the use of H56 or ID93 as therapeutic vaccines (6). These vaccines have numerous advantages such as inexpensive procedure, reliability, safety unlike live attenuated vaccines, and applicability for HIV-infected individuals such as the H1/IC31 candidate vaccine (12). Nevertheless, these candidates have numerous challenges for recipients. These candidates have numerous challenges such as deficiency of stable antigenicity during formulation, storage, repetitive injection in several stages, or lack of enough volunteer population for participation in efficacy evaluation tests $(4-7,13)$.

In summary, in spite of advancement in diagnosis and treatment, tuberculosis remains a major challenge for public health. Nowadays, vaccination is one of the best strategies in the prevention of tuberculosis in epidemic areas. The BCG vaccine as the only available tuberculosis vaccine has several shortcomings such as side effects and low efficacy against both adult pulmonary and latent tuberculosis infections; it is also not recommended for HIV patients. Therefore, several tuberculosis candidate vaccines are under clinical trial experiments. Fusion peptides are 


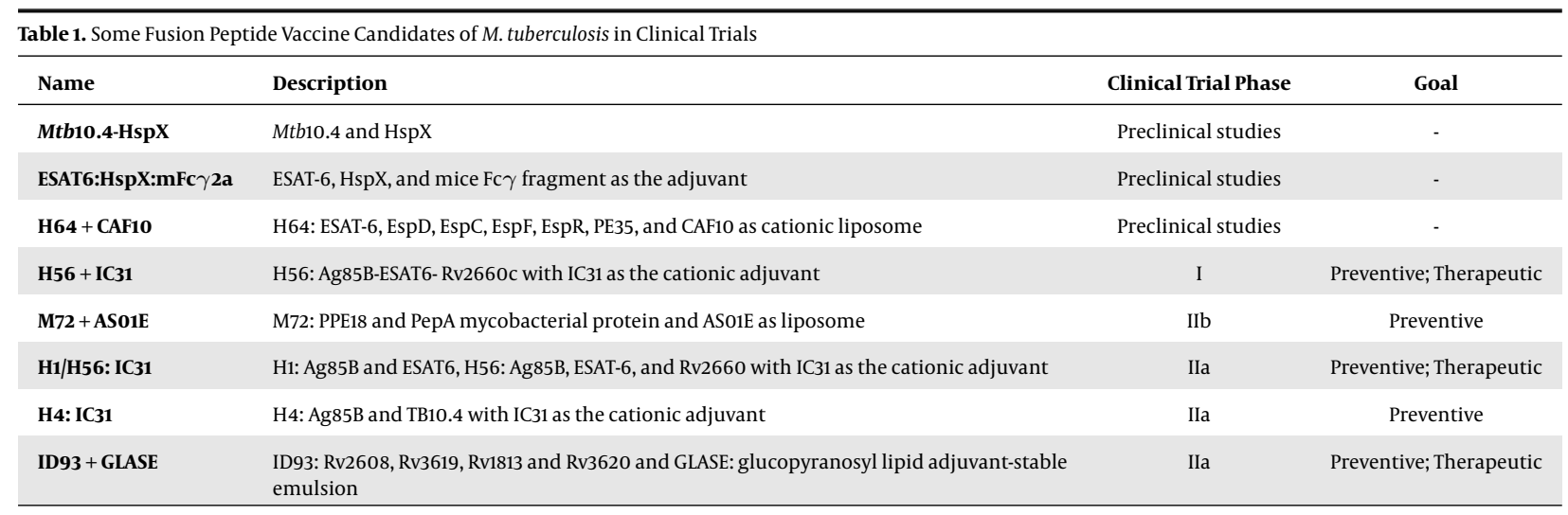

the best option for tuberculosis vaccine production based on animal studies and clinical trials on healthy and TBinfected volunteers.

\section{Footnotes}

Conflicts of Interests: None to declare.

Ethical Approval: The Ethics Committee of Mashhad University of Medical Sciences approved the study.

Funding/Support: The manuscript was not received financial support.

\section{References}

1. World Health Organization. Global tuberculosis report 2016. World Health Organization; 2016.

2. World Health Organization. Global tuberculosis report 2017. World Health Organization; 2017.

3. Getahun H, Matteelli A, Chaisson RE, Raviglione M. Latent Mycobacterium tuberculosis infection. N Engl J Med. 2015;372(22):2127-35. doi: 10.1056/NEJMra1405427. [PubMed: 26017823].

4. Khoshnood S, Heidary M, Haeili M, Drancourt M, Darban-Sarokhalil D, Nasiri MJ, et al. Novel vaccine candidates against Mycobacterium tuberculosis. Int J Biol Macromol. 2018;120(Pt A):180-8. doi: 10.1016/j.ijbiomac.2018.08.037. [PubMed: 30098365].

5. Khademi F, Derakhshan M, Yousefi-Avarvand A, Tafaghodi M, Soleimanpour S. Multi-stage subunit vaccines against Mycobacterium tuberculosis: An alternative to the BCG vaccine or a BCG-prime boost? Expert Rev Vaccines. 2018;17(1):31-44. doi: 10.1080/14760584.2018.1406309. [PubMed: 29148853].
6. Andersen P, Kaufmann SH. Novel vaccination strategies against tuberculosis. Cold Spring Harb Perspect Med. 2014;4(6). doi: 10.1101/cshperspect.a018523. [PubMed: 24890836]. [PubMed Central: PMC4031959].

7. Kaufmann SH. Tuberculosis vaccines: Time to think about the next generation. Semin Immunol. 2013;25(2):172-81. doi: 10.1016/j.smim.2013.04.006. [PubMed: 23706597].

8. Lindenstrom T, Agger EM, Korsholm KS, Darrah PA, Aagaard C, Seder RA, et al. Tuberculosis subunit vaccination provides long-term protective immunity characterized by multifunctional CD4 memory T cells. J Immunol. 2009;182(12):8047-55. doi: 10.4049/jimmunol.0801592. [PubMed: 19494330].

9. Aagaard C, Hoang T, Dietrich J, Cardona PJ, Izzo A, Dolganov G, et al. A multistage tuberculosis vaccine that confers efficient protection before and after exposure. Nat Med. 2011;17(2):189-94. doi: 10.1038/nm.2285. [PubMed: 21258338].

10. Karbalaei Zadeh Babaki M, Soleimanpour S, Rezaee SA. Antigen 85 complex as a powerful Mycobacterium tuberculosis immunogene: Biology, immune-pathogenicity, applications in diagnosis, and vaccine design. Microb Pathog. 2017;112:20-9. doi: 10.1016/j.micpath.2017.08.040. [PubMed: 28942172].

11. Kaufmann SH, Weiner J, von Reyn CF. Novel approaches to tuberculosis vaccine development. Int J Infect Dis. 2017;56:263-7. doi: 10.1016/j.ijid.2016.10.018. [PubMed: 27816661].

12. Reither K, Katsoulis L, Beattie T, Gardiner N, Lenz N, Said K, et al. Safety and immunogenicity of H1/IC31(R), an adjuvanted TB subunit vaccine, in HIV-infected adults with CD4+ lymphocyte counts greater than 350 cells/mm3: A phase II, multi-centre, double-blind, randomized, placebo-controlled trial. PLoS One. 2014;9(12). e114602. doi: 10.1371/journal.pone.0114602. [PubMed: 25490675]. [PubMed Central: PMC4260867].

13. Keikha M, Moghim S, Fazeli H, Nasr-Esfahani B. The fusion multistage synthetic peptides as the best candidates for new tuberculosis vaccine. Adv Biomed Res. 2018;7:122. doi: 10.4103/abr.abr_116_18.[PubMed: 30211135]. [PubMed Central: PMC6124223]. 